\author{
LINEAR AND NON-LINEAR THEORY OF \\ GENERALIZED FUNCTIONS AND ITS APPLICATIONS \\ BANACH CENTER PUBLICATIONS, VOLUME 88 \\ INSTITUTE OF MATHEMATICS \\ POLISH ACADEMY OF SCIENCES \\ WARSZAWA 2010
}

\title{
EQUATIONS IN DIFFERENTIALS IN THE ALGEBRA OF GENERALIZED STOCHASTIC PROCESSES
}

\author{
NADZEYA V. BEDZIUK \\ Belarusian State University \\ Nezavisimosti 4, 220030 Minsk, Belarus \\ E-mail: nbedyuk@gmail.com \\ ALEH L. YABLONSKI \\ Belarusian State University \\ Nezavisimosti 4, 220030 Minsk, Belarus \\ E-mail: yablonski@bsu.by,yablonski_oleg@rambler.ru
}

\begin{abstract}
We consider an ordinary or stochastic nonlinear equation with generalized coefficients as an equation in differentials in the algebra of new generalized functions in the sense of $[8$. Consequently, the solution of such an equation is a new generalized function. We formulate conditions under which the solution of a given equation in the algebra of new generalized functions is associated with an ordinary function or process. Moreover the class of all possible associated functions and processes is described.
\end{abstract}

1. Introduction. The theory of generalized functions is one of the most powerful tools for investigating linear differential equations. However, the distribution theory from the very beginning has an essential disadvantage: it is inapplicable to solutions of nonlinear problems. Therefore, various interpretations of solutions of nonlinear differential equations were proposed by some mathematicians. Unfortunately, different interpretations of the same equation lead, in general, to different solutions; see e.g. $[2,4,6$, $11,14,18]$. Usually, differential equations are used to describe the dynamics of real systems or phenomena. In order to choose an adequate interpretation of such equations one has to consider reasons that are used for modelling the dynamics of the real systems.

2000 Mathematics Subject Classification: Primary 34A37, 60H20; Secondary 46F30, 60G20.

Key words and phrases: algebra of generalized functions and stochastic processes, associated solutions, differential equations with generalized coefficients.

The paper is in final form and no version of it will be published elsewhere. 
In this paper we will consider the following nonlinear equation with generalized coefficients

$$
\dot{X}(t)=f(t, X(t)) \dot{L}(t),
$$

where $t \in[a ; b] \subset \mathbb{R}$ and $\dot{L}(t)$ is the derivative in the distributional sense. In general, since $\dot{L}(t)$ is a distribution and $f(t, X(t))$ is not a smooth function, the product $f(t, X(t)) \dot{L}(t)$ is not well defined and the solution of equation (1) essentially depends on the interpretation. Let us note that if $L$ is a stochastic process then equation (1) is a stochastic differential equation.

We will investigate equation (1) by using the algebra of mnemofunctions (new generalized functions) or mnemoprocesses. It is worth mentioning that the first algebra of new generalized functions were proposed by J.-F. Colombeau in [3. Definitions of other algebras can be found in [5, 16]. The general methods of construction of such algebras were proposed by A. B. Antonevich and Ya. V. Radyno in 11. Analogous algebras for stochastic processes were defined in [8, 12, 13.

In this paper we interpret equation (1) as an equation in differentials in the algebra of new generalized functions or processes from [8]. Such an approach allows us to investigate ordinary and stochastic differential equations from the common ground.

Algebraic interpretation states that the solution of equation $(1)$ is a new generalized function or process. The main purpose of this article is to formulate conditions under which this new generalized function or process is associated with some ordinary function or process which can be naturally called a solution of equation (1). Moreover we will describe all functions and processes which can be solutions of equation (1) in this sense.

2. The algebra of mnemofunctions and mnemoprocesses. In this section we recall the definition of the algebra of new generalized functions and mnemoprocesses from [8], see also [9] and [17.

First we define an extended real line $\widetilde{\mathbb{R}}$ using a construction typical of non-standard analysis. Let $\overline{\mathbb{R}}=\left\{\left\{x_{n}\right\}_{n=1}^{\infty}: x_{n} \in \mathbb{R}\right.$ for all $\left.n \in \mathbb{N}\right\}$ be the set of all real sequences. We will call two sequences $\left\{x_{n}\right\} \in \overline{\mathbb{R}}$ and $\left\{y_{n}\right\} \in \overline{\mathbb{R}}$ equivalent if there is a natural number $N$ such that $x_{n}=y_{n}$ for all natural $n>N$. The set $\widetilde{\mathbb{R}}$ of equivalence classes will be called the extended real line and any of the classes a generalized real number.

It is easy to see that $\mathbb{R} \subset \widetilde{\mathbb{R}}$ as one may associate with any ordinary number $x \in \mathbb{R}$ the class containing the constant sequence corresponding to $x$ given by $x_{n}=x$ for all $n \in \mathbb{N}$. Operations on generalized real numbers are defined typically for non-standard analysis. For instance, the product $\widetilde{x} \widetilde{y}$ of two generalized real numbers is defined as the class of sequences equivalent to the sequence $\left\{x_{n} y_{n}\right\}$, where $\left\{x_{n}\right\}$ and $\left\{y_{n}\right\}$ are arbitrary representatives of the classes $\widetilde{x}$ and $\widetilde{y}$ respectively. It is evident that $\widetilde{\mathbb{R}}$ is an algebra.

For any segment $\mathbf{T}=[a ; b] \subset \mathbb{R}$ one can construct an extended segment $\widetilde{\mathbf{T}}$ in a similar way. Let $H$ denote the subset of $\widetilde{\mathbb{R}}$ of nonnegative "infinitely small numbers":

$$
H=\left\{\widetilde{h} \in \widetilde{\mathbb{R}}: \widetilde{h}=\left[\left\{h_{n}\right\}\right], h_{n}>0 \text { for all } n \in \mathbb{N}, \lim h_{n}=0\right\} .
$$

Let $(\Omega, \mathcal{F}, \mathrm{P})$ be a probability space. Consider the set of sequences of stochastic processes with infinitely differentiable paths $\left\{f_{n}(t, \omega)\right\}$ on $\mathbb{R}$. We will say that two se- 
quences $\left\{f_{n}(t, \omega)\right\}$ and $\left\{g_{n}(t, \omega)\right\}$ are equivalent if there is a natural number $N$ such that $f_{n}(t, \omega)=g_{n}(t, \omega)$ for all natural $n>N, t \in \mathbb{R}$ and $\omega \in \Omega$. The set of classes of equivalent sequences is denoted by $\mathcal{G}(\mathbb{R}, \Omega)$ and its elements are called mnemoprocesses or generalized stochastic processes. Similarly one can define the space $\mathcal{G}(\mathbf{T}, \Omega)$ for any interval $\mathbf{T}=[a ; b]$. If we replace stochastic processes by deterministic smooth functions then we obtain the space $\mathcal{G}(\mathbb{R})$ or $\mathcal{G}(\mathbf{T})$ of mnemofunctions or generalized functions. If we endow all these spaces with natural operations of addition and multiplication then they become algebras.

For each distribution $f$ we can construct a sequence $\left\{f_{n}\right\}$ of smooth functions such that $f_{n}$ converges to $f$ (e.g. one can consider the convolution of $f$ with some $\delta$-sequence). This sequence defines the mnemofunction which corresponds to the distribution $f$. Thus the space of distributions is a subset of the algebra of mnemofunctions. However, in this case, infinitely many mnemofunctions correspond to one distribution (e.g., by taking different $\delta$-sequences). We will say that the mnemofunction (mnemoprocess) $\widetilde{f}=\left[\left\{f_{n}\right\}\right]$ is associated with a function (process) $f$ from some topological space if $f_{n}$ converges to $f$ in this space.

Let $\tilde{f}=\left[\left\{f_{n}\right\}\right]$ and $\widetilde{g}=\left[\left\{g_{n}\right\}\right]$ be generalized functions (processes). Then the composition $\tilde{f} \circ \widetilde{g}$ is defined by $\tilde{f} \circ \widetilde{g}=\left[\left\{f_{n} \circ g_{n}\right\}\right] \in \mathcal{G}(\mathbb{R})$. Similarly, one can define the value of the mnemofunction $\widetilde{f}$ at the generalized real point $\widetilde{x}=\left[\left\{x_{n}\right\}\right] \in \widetilde{\mathbb{R}}$ as $\widetilde{f}(\widetilde{x})=\left[\left\{f_{n}\left(x_{n}\right)\right\}\right]$.

For each $\widetilde{h}=\left[\left\{h_{n}\right\}\right] \in H$ and $\widetilde{f}=\left[\left\{f_{n}\right\}\right] \in \mathcal{G}(\mathbb{R}, \Omega)$ we define a differential $d_{\widetilde{h}} \widetilde{f} \in$ $\mathcal{G}(\mathbb{R}, \Omega)$ by $d_{\widetilde{h}} \tilde{f}(t)=\left[\left\{f_{n}\left(t+h_{n}\right)-f_{n}(t)\right\}\right]$ for $t \in \mathbb{R}$. The construction of the differential was proposed by N. V. Lazakovich (see [8]).

Now we can give an interpretation of equation (1) using the introduced algebras. Let $L(t), t \in[a ; b]=\mathbf{T}$, be a right-continuous function of finite variation (or continuous process). We replace ordinary functions (processes) in equation (1) by the corresponding new generalized functions (processes) and then write differentials in the algebra. So we have

$$
d_{\widetilde{h}} \tilde{X}(\widetilde{t})=\widetilde{f}(\widetilde{t}, \widetilde{X}(\widetilde{t})) d_{\widetilde{h}} \widetilde{L}(\widetilde{t}),
$$

with the initial value $\left.\widetilde{X}\right|_{[\widetilde{a} ; \widetilde{h})}=\widetilde{X}^{0}$, where $\widetilde{h}=\left[\left\{h_{n}\right\}\right] \in H, \widetilde{a}=[\{a\}] \in \widetilde{\mathbf{T}}, \widetilde{t}=\left[\left\{t_{n}\right\}\right] \in \widetilde{\mathbf{T}}$, $\widetilde{X}=\left[\left\{X_{n}\right\}\right], \tilde{f}=\left[\left\{f_{n}\right\}\right], \widetilde{X}^{0}=\left[\left\{X_{n}^{0}\right\}\right]$, and $\widetilde{L}=\left[\left\{L_{n}\right\}\right]$ are elements of $\mathcal{G}(\mathbb{R})($ or $\mathcal{G}(\mathbb{R}, \Omega)$ for stochastic equations). Moreover $\widetilde{f}$ and $\widetilde{L}$ are associated with $f$ and $L$ respectively. If $\widetilde{X}$ is associated with some function (process) $X$ then we say that $X$ is a solution of equation (1).

The following theorem from [9] gives necessary and sufficient conditions for existence and uniqueness of solutions of equation $(2)$.

THEOREM 2.1. Equation (2) with the initial condition $\left.\widetilde{X}\right|_{[\widetilde{a} ; \tilde{h})}=\widetilde{X}^{0}$ admits a solution if and only if the following equality holds for some representatives $\left\{f_{n}\right\} \in \widetilde{f},\left\{L_{n}\right\} \in \widetilde{L}$, $\left\{X_{n}^{0}\right\} \in \widetilde{X}^{0}$, for all sufficiently large $n \in \mathbb{N}$ and for all $l=0,1, \ldots$ :

$$
\lim _{t \rightarrow h_{n}-0} \frac{d^{l}}{d t^{l}} X_{n}^{0}(t)=\lim _{t \rightarrow+0} \frac{d^{l}}{d t^{l}}\left(X_{n}^{0}(t)+f_{n}\left(t, X_{n}^{0}(t)\right)\left(L_{n}\left(t+h_{n}\right)-L_{n}(t)\right)\right) .
$$

If a solution exists then it is unique. 
The purpose of the present paper is to investigate when the solution $\widetilde{X}$ of equation 2 is associated with some function (process) and to describe all possible associated solutions.

3. Ordinary differential equations. In this section we will formulate main results in case of a non-continuous deterministic function $L$.

Let $L(t), t \in \mathbf{T}=[a ; b]$, be a right-continuous function of finite variation. We will assume that $L(t)=L(b)$ if $t>b$ and $L(t)=L(a)$ if $t<a$. Denote by $V_{u}^{v} L$ the total variation of the function $L$ on the interval $[u ; v] \subset \mathbf{T}$. Suppose that $f$ is a Lipschitz continuous function with a constant $C$ and for all $x \in \mathbb{R}$ and $t \in \mathbb{T}$ :

$$
|f(t, x)| \leq C(1+|x|) .
$$

Consider the following convolutions with a $\delta$-sequence $\left\{\rho_{n}\right\}$ as representatives of mnemofunction $\widetilde{L}$ from equation 22 :

$$
L_{n}(t)=\left(L * \rho_{n}\right)(t)=\int_{0}^{1 / n} L(t+s) \rho_{n}(s) d s,
$$

where $\rho_{n} \in C^{\infty}(\mathbb{R}), \rho_{n} \geq 0, \operatorname{supp} \rho_{n} \subseteq[0 ; 1 / n]$, and $\int_{0}^{1 / n} \rho_{n}(s) d s=1$ for $n \in \mathbb{N}$.

In the same way we set

$$
f_{n}(t, x)=\left(f * \widetilde{\rho}_{n}\right)(t, x)=\int_{[0,1 / n]^{2}} f(t+u, x+v) \widetilde{\rho}_{n}(u, v) d u d v,
$$

where $\widetilde{\rho}_{n} \in C^{\infty}\left(\mathbb{R}^{2}\right), \widetilde{\rho}_{n} \geq 0$, supp $\widetilde{\rho}_{n} \subseteq[0,1 / n]^{2}$, and $\int_{[0,1 / n]^{2}} \widetilde{\rho}_{n}(u, v) d u d v=1$ for $n \in \mathbb{N}$.

By using representatives we can rewrite equation (2) in the following form:

$$
\left\{\begin{array}{l}
X_{n}\left(t+h_{n}\right)-X_{n}(t)=f_{n}\left(t, X_{n}(t)\right)\left(L_{n}\left(t+h_{n}\right)-L_{n}(t)\right), \\
\left.X_{n}\right|_{\left[a ; a+h_{n}\right)}(t)=X_{n}^{0}(t) .
\end{array}\right.
$$

The solution $\tilde{X}$ of equation (2) is associated with some function if and only if the sequence $\left\{X_{n}\right\}$ of the solutions of equation (6) converges. Therefore we have to investigate the limiting behavior of the sequence $\left\{X_{n}\right\}$.

Let $t$ be an arbitrary point of $\mathbf{T}$. There exist $m_{t} \in \mathbb{N}$ and $\tau_{t} \in\left[a ; a+h_{n}\right)$ such that $t=\tau_{t}+m_{t} h_{n}$. Set $t_{k}=\tau_{t}+k h_{n}$ for $k=0,1, \ldots, m_{t}$. Then the solution of equation (6) can be written as

$$
X_{n}(t)=X_{n}^{0}\left(\tau_{t}\right)+\sum_{k=0}^{m_{t}-1} f_{n}\left(t_{k}, X_{n}\left(t_{k}\right)\right)\left(L_{n}\left(t_{k+1}\right)-L_{n}\left(t_{k}\right)\right) .
$$

Consider the function $F_{n}(x):[-\infty ;+\infty] \rightarrow[0 ; 1]$ given by

$$
F_{n}(x)=\int_{x}^{1 / n} \rho_{n}(s) d s .
$$

Since $\rho_{n}(s) \geq 0$, then $F_{n}$ is a non-increasing function, $0 \leq F_{n}(x) \leq 1$ and $F_{n}(+\infty)=0$, $F_{n}(-\infty)=1$. Denote by $F_{n}^{-1}$ the inverse function of $F_{n}$, i.e., $F_{n}^{-1}:[0 ; 1] \rightarrow[-\infty ;+\infty]$ and

$$
F_{n}^{-1}(u)=\sup \left\{x: F_{n}(x)=u\right\} .
$$


In order to describe the limits of the sequence $\left\{X_{n}\right\}$ we consider the integral equation

$$
X(t)=x^{0}+\int_{a}^{t} f(s, X(s)) d L^{c}(s)+\sum_{a<s \leq t}(\varphi(\Delta L(s) f, X(s-), 1)-X(s-)),
$$

where $t \in \mathbf{T}, L^{c}$ is the continuous part of the function $L, \Delta L(s)=L(s+)-L(s-)$ is the size of the jump of the function $L$ at $s, \varphi(z, x, u)$ is the solution of the integral equation

$$
\varphi(z, x, u)=x+\int_{[0 ; u)} z(\varphi(z, x, v)) \mu(d v),
$$

and $\mu$ is a probability measure defined on the Borel subsets of the interval $[0 ; 1]$.

As was shown in [17 there exists a unique solution of equation (7) if $f$ is a Lipschitz continuous function.

Definition 3.1. We say that a function $\sigma:[0 ; 1] \rightarrow[0 ; 1]$ belongs to class $\mathbf{G}$ if there is a system of pairwise-disjoint intervals $\left(a_{i} ; b_{i}\right] \subseteq[0 ; 1], i \in I$, such that

$$
\sigma(u)= \begin{cases}b_{i}, & u \in\left(a_{i} ; b_{i}\right] \\ u, & u \notin \bigcup_{i \in I}\left(a_{i} ; b_{i}\right] .\end{cases}
$$

Notice that every $\sigma \in \mathbf{G}$ is a non-decreasing and right-continuous function, so it uniquely generates a probability measure defined on the Borel subsets of $[0 ; 1]$.

The following theorems describe the limits of the sequence $\left\{X_{n}\right\}$.

THEOREM 3.2. Let $f$ be a Lipschitz function satisfying (3) and $L$ be a right-continuous function of finite variation. Suppose that $\int_{t \in \mathbf{T}}\left|X_{n}^{0}\left(\tau_{t}\right)-x^{0}\right| d t \rightarrow 0$ and, for a certain function $\sigma:[0 ; 1] \rightarrow[0 ; 1]$, we have $F_{n}\left(F_{n}^{-1}(u)-\delta h_{n}\right) \rightarrow \sigma(u)$ as $n \rightarrow \infty$ and $h_{n} \rightarrow 0$ for all $\delta \in(0 ; 1)$ and for all continuity points $u \in[0,1]$ of $\sigma$. Then $\sigma$ belongs to $\mathbf{G}$ and

$$
\int_{\mathbf{T}}\left|X_{n}(t)-X(t)\right| d t \rightarrow 0
$$

as $n \rightarrow \infty$ and $h_{n} \rightarrow 0$, where $X_{n}$ is the solution of equation (6) and $X$ is the solution of equation (7) with $\varphi$ being the solution of (8) for the measure $\mu$ generated by $\sigma$.

THEOREM 3.3. Let $L$ be a right-continuous function of finite variation. Suppose that, for each Lipschitz function $f$ satisfying (3), the solution $X_{n}$ of equation (6) converges in $\mathbf{L}_{\mathbf{1}}(\mathbf{T})$ as $n \rightarrow \infty$ and $h_{n} \rightarrow 0$. If the function $L$ is continuous, then the limit of $X_{n}$ is the solution of equation (7). If $L$ is discontinuous, then there exists a function $\sigma \in \mathbf{G}$ such that $F_{n}\left(F_{n}^{-1}(u)-\delta h_{n}\right) \rightarrow \sigma(u)$ as $n \rightarrow \infty$ and $h_{n} \rightarrow 0$ for all $\delta \in(0,1)$ and for all continuity points $u \in[0,1]$ of $\sigma$, and the limit of $X_{n}$ is the solution of equation (7) with $\varphi$ being the solution of (8) for the measure $\mu$ generated by $\sigma$.

4. Stochastic differential equations. In this section we will formulate main results in case $L$ is a continuous stochastic process.

Let $B(t), t \in \mathbf{T}$, be a one-dimensional standard process of Brownian motion. If we put $L=B$ in equation (1) then we obtain a differential equation with generalized stochastic coefficients. It can be interpreted as a stochastic differential equation. Unfortunately, the solution of the stochastic equation essentially depends on the stochastic integral which 
is used in the equation. Usually mathematicians use the Itô integral and practicians use the Stratonovich integral.

Let us recall the definition of the $\theta$-integral which is a generalization of the Itô and Stratonovich integrals (see e.g. [15]). Suppose that $\theta \in[0 ; 1]$ and $Z(t)$ is a stochastic process. Let $t \in \mathbf{T}$ and $0=t_{0}<t_{1}<\cdots<t_{m}=t$ be a partition of $[0 ; t]$. Set $|\sigma|=$ $\max _{0 \leq k \leq m-1}\left|t_{k+1}-t_{k}\right|$. If, for any $t \in \mathbf{T}$, the limit in the formula

$$
(\theta) \int_{0}^{t} X(s) d B(s)=\lim _{|\sigma| \rightarrow 0} \sum_{k=0}^{m-1}\left(\theta X\left(t_{k+1}\right)+(1-\theta) X\left(t_{k}\right)\right)\left(B\left(t_{k+1}\right)-B\left(t_{k}\right)\right)
$$

exists and does not depend on partitions, then the $\theta$-integral of $X$ exists and is defined by this formula. For $\theta=0$ we obtain the Itô integral and for $\theta=1 / 2$ it coincides with the Stratonovich integral.

As in the deterministic case we interpret equation (1) as an equation in differentials in the algebra of new generalized processes. Proceeding as in the previous section we rewrite equation (2) in the following form:

$$
\left\{\begin{array}{l}
X_{n}\left(t+h_{n}\right)-X_{n}(t)=f_{n}\left(t, X_{n}(t)\right)\left(B_{n}\left(t+h_{n}\right)-B_{n}(t)\right), \\
\left.X_{n}\right|_{\left[a ; a+h_{n}\right)}(t)=X_{n}^{0}(t) .
\end{array}\right.
$$

Here $f_{n}$ is the convolution of a given Lipschitz function $f$ with a $\delta$-sequence $\left\{\widetilde{\rho}_{n}\right\}$ as defined above and $B_{n}$ is the convolution of $B$ with $\rho_{n}$.

As in the deterministic case the solution $\widetilde{X}$ of equation 2 is associated with some process if and only if the sequence of the solutions $X_{n}$ of equation $(9)$ converges.

We will characterize limiting behavior of $X$ by using the sequence $\left\{K\left(n, h_{n}\right)\right\}$ of the following functions:

$$
K\left(n, h_{n}\right)=\iint_{\substack{0 \leq u, v \leq 1 / n \\|u-v| \leq h_{n}}}\left(1-\frac{|u-v|}{h_{n}}\right) \rho_{n}(u) \rho_{n}(v) d u d v .
$$

It is evident that $0 \leq K\left(n, h_{n}\right) \leq 1$.

In order to describe possible limits of $X_{n}$ we consider the following stochastic equation:

$$
X(t)=x^{0}+(\theta) \int_{0}^{t} f(s, X(s)) d B(s), \quad t \in T .
$$

For functions $f \in \mathbf{C}_{b}^{2}(\mathbf{T}, \mathbb{R})$ there exist unique (strong) solutions of equation (10) (see e.g. 15 or [7).

The following theorems give a complete description of the limiting behavior of the sequence $\left\{X_{n}\right\}$.

Theorem 4.1. Suppose that $f \in \mathbf{C}_{b}^{2}(\mathbf{T}, \mathbb{R}), f \not \equiv$ const, $n^{2} h_{n} \rightarrow \infty$, and

$$
\sup _{t \in\left[0, h_{n}\right)} \mathrm{E}\left[X_{n}^{0}(t)-x^{0}\right]^{2} \rightarrow 0
$$

as $n \rightarrow \infty$ and $h_{n} \rightarrow 0$. Then the solution $X_{n}$ of equation (9) converges in $\mathbf{L}^{2}(\Omega, \mathcal{F}, \mathrm{P})$ and uniformly in $t \in \mathbf{T}$ if and only if the numerical sequence $\left\{K\left(n, h_{n}\right)\right\}$ converges. 
Theorem 4.2. Suppose that $f \in \mathbf{C}_{b}^{2}(\mathbf{T}, \mathbb{R}), \theta \in[0 ; 1 / 2], K\left(n, h_{n}\right) \rightarrow(1-2 \theta), n^{2} h_{n} \rightarrow \infty$, and

$$
\sup _{t \in\left[0, h_{n}\right)} \mathrm{E}\left[X_{n}^{0}(t)-x^{0}\right]^{2} \rightarrow 0
$$

as $n \rightarrow \infty$ and $h_{n} \rightarrow 0$. Then

$$
\sup _{t \in \mathbf{T}} \mathrm{E}\left[X_{n}(t)-X(t)\right]^{2} \rightarrow 0
$$

where $X_{n}$ is the solution of equation (9) and $X$ is the solution of equation (10).

5. Associated solutions. In this section we describe associated solutions of equation (2) in the deterministic and stochastic cases. In the deterministic case we have

THEOREM 5.1. Let $f$ be a Lipschitz function satisfying (3) and $L$ be a right-continuous function of finite variation. Suppose that for representatives of mnemofunctions $\tilde{f}$ and $\widetilde{L}$ given by formulae (5) and (4) respectively conditions of Theorem 2.1 hold. Then the solution $\widetilde{X}$ of equation (2) is associated with $X$ if and only if $X$ satisfies equation (7) with some probability measure $\mu$ generated by a function from the class $\mathbf{G}$.

REMARK 5.2. Let us note that in order to obtain other associated solutions of equation (2) one has to consider other types of representatives $f_{n}$ and $L_{n}$.

In the stochastic case the following result holds:

THEOREM 5.3. Let $f \in \mathbf{C}_{b}^{2}(\mathbf{T}, \mathbb{R})$ and $L$ be a Brownian motion. Suppose that for representatives of $\tilde{f}$ and $\widetilde{L}$ given by formulae (5) and (4) respectively the conditions of Theorem 2.1 hold. Then the solution $\widetilde{X}$ of equation 2) is associated with $X$ if and only if $X$ satisfies equation (10) for some $\theta \in[0 ; 1 / 2]$.

REMARK 5.4. If $\theta \in[1 / 2 ; 1]$ then the solution of equation 10 is the associated solution of equation 22 with the "backward" differential $d_{\widetilde{h}} f$ given by $d_{\widetilde{h}} \widetilde{f}(t)=\left[\left\{f_{n}(t)-f_{n}\left(t-h_{n}\right)\right\}\right]$ for $t \in \mathbb{R}$; see [10] for details.

\section{References}

[1] A. B. Antonevich and Ya. V. Radyno, On a general method for constructing algebras of generalized functions, Sov. Math. Dokl. 43 (1991), 680-684.

[2] P. Antosik and J. Ligęza, Products of measures and functions of finite variations, in: Generalized Functions and Operational Calculus, Proc. Conf., Varna 1975, (1979), 20-26.

[3] J. F. Colombeau, A multiplication of distributions, J. Math. Anal. Appl. 94 (1983), 96-115.

[4] P. C. Das and R. R. Sharma, Existence and stability of measure differential equations, Czechoslovak Math. J. 22 (1972), 145-158.

[5] Yu. V. Egorov, A contribution to the theory of generalized functions, Russian Math. Surveys 45 (1990), 1-49.

[6] A. F. Filippov, Differential Equations with Discontinuous Right Hand Sides, Mathematics and its Applications (Soviet Series) 18, Kluwer, Dordrecht, 1988.

[7] N. Ikeda and S. Watanabe, Stochastic Differential Equations and Diffusion Processes, Amsterdam-Oxford-New York, 1981. 
[8] N. V. Lazakovich, Stochastic differentials in the algebra of generalized random processes, Dokl. Akad. Nauk Belarusi 38 (1994), no. 5, 23-27 (in Russian).

[9] N. V. Lazakovich, S. P. Stashulenok, and I. V. Yufereva, Stochastic differential equations in the algebra of generalized random processes, Differ. Equ. 31 (1995), 2056-2058.

[10] N. V. Lazakovich and A. L. Yablonski, On the approximation of the solutions of stochastic equations with $\theta$-integrals, Stochastics and Stochastics Reports 76 (2004), 135-145.

[11] J. Ligęza, On generalized solutions of some differential nonlinear equations of order $n$, Ann. Polon. Math. 31 (1975), 115-120.

[12] Z. Lozanov-Crvenković and S. Pilipović, Some classes of Colombeau's generalized random processes, Novi Sad J. Math. 27 (1997), 133-143.

[13] M. Oberguggenberger, Generalized functions and stochastic processes, Progr. Probab. 36 (1995), 215-229.

[14] S. G. Pandit and S. G. Deo, Differential Systems Involving Impulses, Lect. Notes in Math. 954, Springer, Berlin, 1982.

[15] V. S. Pugachev and I. N. Sinitsyn, Stochastic Differential Systems. Analysis and Filtering, Wiley, Chichester, 1987.

[16] E. E. Rosinger, Generalized Solutions of Nonlinear Partial Differential Equations, NorthHolland, Amsterdam, 1987.

[17] A. L. Yablonski, Differential equations with generalized coefficients, Nonlinear Anal. 63 (2005), 171-197.

[18] S. T. Zavalishchin and A. N. Sesekin, Dynamic Impulse Systems. Theory and Applications, Mathematics and its Applications 394, Kluwer, Dordrecht, 1997. 